\title{
THE DISTRIBUTION OF ABSOLUTELY IRREDUCIBLE POLYNOMIALS IN SEVERAL INDETERMINATES
}

\author{
M. L. FREDMAN
}

\begin{abstract}
Asymptotic formulas are derived for the distribution of absolutely irreducible polynomials in two indeterminates over finite fields. A pair of inversion formulas is presented which yield exact formulas relating the distributions of irreducible and absolutely irreducible polynomials.
\end{abstract}

1. Introduction. An absolutely irreducible polynomial over a field $F$ is a polynomial that has no proper factorization in any extension field of $F$. The only absolutely irreducible polynomials in one indeterminate are first degree polynomials. In this paper we examine the distribution of absolutely irreducible polynomials in two indeterminates with coefficients in GF $(q)$. L. Carlitz has derived ([1] and [2]) estimates for the number of irreducible polynomials in two indeterminates with coefficients in $\mathrm{GF}(q)$. We use his estimates to derive some of the results presented here.

Let $f_{j}(m, n)$ denote the number of normalized polynomials in $x$ and $y$ with coefficients in $\operatorname{GF}\left(q^{j}\right)$, where $m$ is the degree in $x$ and $n$ is the degree in $y$. Let $\psi_{j}(m, n)$ denote the number of normalized irreducible polynomials. Finally, let $\tau_{j}(m, n)$ denote the number of normalized absolutely irreducible polynomials with coefficients contained in $\operatorname{GF}\left(q^{j}\right)$ but not contained in any proper subfield of $\operatorname{GF}\left(q^{j}\right)$ that contains $\operatorname{GF}(q)$. We prove

$$
\tau_{j}(m, n)=\psi_{j}(m, n)+O\left(q^{j(m+2)(n+2) / 2}\right), \quad m, n>0 .
$$

Combining this with the result in [2], we have

$$
\tau_{j}(m, n) \sim\left(1-q^{-j m}\right) f_{j}(m, n) \quad(n \rightarrow \infty)
$$

for fixed $m$.

We remark that $\tau_{1}(m, n)$ is the number of normalized absolutely irreducible polynomials with coefficients in $\mathrm{GF}(q)$.

2. The relation between $\tau_{j}(m, n)$ and $\psi_{j}(m, n)$. We begin by presenting the following formula.

Received by the editors June $29,1970$.

AMS 1970 subject classifications. Primary 12C05; Secondary 10A20.

Key words and phrases. Irreducible polynomial, absolutely irreducible, several indeterminates, finite fields, inversion formulas, distributions.

(C) American Mathematical Society 1972 
THEOREM 1.

$$
\psi_{j}(m, n)=\sum_{d|m ; d| n}(1 / d) \sum_{e \mid j ;(e, d)=1} \tau_{j d / e}(m / d, n / d) .
$$

Proof. Let $p(x, y)$ be an irreducible polynomial with coefficients in $\mathrm{GF}\left(q^{j}\right)$ of degrees $m$ and $n$ in $x$ and $y$. Assume $p(x, y)=t_{1}(x, y) t_{2}(x, y) \cdots$ $t_{d}(x, y)$ when factored into absolute irreducibles. It is not difficult to show

(A) The coefficients of each polynomial, $t_{1}(x, y), \cdots, t_{d}(x, y)$, are contained in $\mathrm{GF}\left(q^{j d}\right)$ but in no smaller field containing $\mathrm{GF}\left(q^{j}\right)$.

(B) $t_{1}(x, y), \cdots, t_{d}(x, y)$ are distinct and they form the complete set of automorphic images of $t_{1}(x, y)$ with respect to the automorphisms of $\mathrm{GF}\left(q^{j d}\right)$ that fix $\mathrm{GF}\left(q^{j}\right)$.

Conversely, if $t_{1}(x, y), \cdots, t_{d}(x, y)$ satisfy (A) and (B), then $t_{1}(x, y) t_{2}(x, y) \cdots t_{d}(x, y)$ is irreducible in $\mathrm{GF}\left(q^{j}\right)$. Hence,

$$
\psi_{j}(m, n)=\sum_{d|m ; d| n}(1 / d) H_{j, d}(m / d, n / d)
$$

where $H_{j, d}(m, n)$ denotes the number of normalized absolutely irreducible polynomials with coefficients contained in $\operatorname{GF}\left(q^{j d}\right)$ but in no smaller field containing $\mathrm{GF}\left(q^{j}\right)$. Clearly,

$$
H_{j, d}(m, n)=\sum_{r: \operatorname{lcm}(r, j)=d j} \tau_{r}(m, n)=\sum_{e \mid j ;(e, d)=1} \tau_{j d / e}(m, n) .
$$

Equations (4) and (5) imply (3), and this proves the theorem.

It is convenient to rewrite equation (3) as follows:

where

$$
\psi_{j}(m, n)-S_{j}(m, n)=\sum_{\left.e\right|^{j}} \tau_{e}(m, n)
$$

$$
S_{j}(m, n)=\sum_{e|j d| m: d \mid n ; d \neq 1 ;(d, e)=1}(1 / d) \tau_{j d / e}(m / d, n / d) .
$$

Using (6) and (6.1), $\tau_{j}(m, n)$ can be computed recursively in terms of $\psi_{j}(m, n)$. When $(m, n)=1$, we clearly have $S_{j}(m, n)=0$. Inverting (6), we obtain $\tau_{j}(m, n)=\sum_{d \mid j} \mu(j / d) \psi_{d}(m, n)$ when $(m, n)=1$. Now assume $\tau_{j}(a, b)$ has been computed when $(a, b)<k$, and assume $(m, n)=k$. Then we have enough information to compute $S_{j}(m, n)$ using (6.1); and by inverting (6) we can compute $\tau_{j}(m, n)$.

3. Estimates for $\tau_{j}(m, n)$. We are now ready to prove (1).

THEOREM 2. $\tau_{j}(m, n)=\psi_{j}(m, n)+O\left(q^{j(m+2)(n+2) / 2}\right), m, n>0$.

Proof. Referring to equations (6) and (6.1), we begin by showing that $S_{j}(m, n)=O\left(q^{j(m+2)(n+2) / 2}\right)$. Clearly $\tau_{j}(m, n) \leqq f_{j}(m, n) \leqq q^{j(m+1)(n+1)}$. Hence,

$$
\tau_{j d / e}(m / d, n / d)=O\left(q^{(j d / e)(m / d+1)(n / d+1)}\right),
$$


and

Finally,

$$
\sum_{d|m ; d| n ; d \neq 1}(1 / d) \tau_{j d / e}(m / d, n / d)=O\left(q^{(j / e)(m+2)(n+2) / 2}\right)
$$

$$
S_{j}(m, n)=\sum_{e \mid j} O\left(q^{(j / e)(m+2)(n+2) / 2}\right)=O\left(q^{j(m+2)(n+2) / 2}\right) .
$$

Using this estimate for $S_{j}(m, n)$, we invert (6) to obtain

Hence

$$
\tau_{j}(m, n)=\sum_{d \mid j} \mu(j / d) \psi_{d}(m, n)+\sum_{d \mid j} O\left(q^{d(m+2)(n+2) / 2}\right) .
$$

Now

$$
\tau_{j}(m, n)=\sum_{d \mid j} \mu(d) \psi_{j / d}(m, n)+O\left(q^{j(m+2)(n+2) / 2}\right)
$$

$$
\sum_{d \mid j} \mu(j / d) \psi_{d}(m, n)=\psi_{j}(m, n)+\sum_{d \mid j ; d \neq 1} \mu(d) \psi_{j / d}(m, n) .
$$

Using the fact that $\psi_{j / d}(m, n) \leqq f_{j / d}(m, n)=O\left(q^{(j / d)(m+1)(n+1)}\right)$, we have

$$
\sum_{d \mid j} \mu(d) \psi_{j / d}(m, n)=\psi_{j}(m, n)+O\left(q^{j(m+1)(n+1) / 2}\right) .
$$

Theorem 2 is proved by combining equations (7) and (8).

THEOREM 3. $\tau_{j}(m, n) \sim\left(1-q^{-j m}\right) f_{j}(m, n)(n \rightarrow \infty)$ for fixed $m$.

Proof. It is easy to show that $f_{j}(m, n)>A_{j} q^{j(m+1)(n+1)}$ for some constant $A_{j}>0$. From Theorem 2 it follows that $\tau_{j}(m, n)=\psi_{j}(m, n)+$ $o\left(f_{j}(m, n)\right)(n \rightarrow \infty)$ for fixed $m>0$. In [2], Carlitz proves that $\psi_{j}(m, n) \sim$ $\left(1-q^{-j m}\right) f_{j}(m, n)(n \rightarrow \infty)$ for fixed $m$. These considerations prove the theorem for the case where $m>0$. When $m=0,\left(1-q^{-j m}\right)=0$, and $\tau_{j}(m, n)=0$ when $n>1$. Hence, the theorem is obvious for this case.

We conclude this paper with some remarks about the single indeterminate analogue of equation (3):

$$
\psi_{j}(m)=\sum_{d \mid m}(1 / d) \sum_{e \mid j ;(e, d)=1} \tau_{j d / e}(m / d) .
$$

It is an interesting exercise to derive the formula,

$$
\psi_{j}(m)=(1 / m) \sum_{d \mid m} \mu(d) q^{j m / d},
$$

from considerations involving equation (9). First, it is clear that $\tau_{j}(m)=0$ when $m>1$. Recalling the definition of $\tau_{j}(m)$, it is easy to show that $q^{j}=\sum_{d \mid j} \tau_{d}(1)$. Hence, $\tau_{j}(1)=\sum_{d \mid j} \mu(d) q^{j / d}$. Substituting these expressions for $\tau_{j}(m)$ in equation (9), we obtain

$$
\psi_{j}(m)=(1 / m) \sum_{e \mid j ;(e, m)=1} \sum_{d \mid j m / e} \mu(d) q^{j m / d e} .
$$


Now in equation (10), either $d=d_{1} d_{2}$ where $\left(d_{1}, m\right)=1, d_{1}|j| e, d_{2} \mid m$; or $\mu(d)=0$. Hence,

$$
\begin{aligned}
\psi_{j}(m) & =(1 / m) \sum_{e \mid j ;(e, m)=1} \sum_{d_{1} \mid j / e:\left(d_{1}, m\right)=1} \mu\left(d_{1}\right) \sum_{d_{2} \mid m} \mu\left(d_{2}\right) q^{j m /\left(d_{1} d_{2} e\right)} \\
& =(1 / m) \sum_{r \mid j ;(r, m)=1} \sum_{d_{1} e=r} \mu\left(d_{1}\right)\left(\sum_{d_{2} \mid m} \mu\left(d_{2}\right) q^{j m / r d_{2}}\right)=(1 / m) \sum_{d \mid m} \mu(d) q^{j m / d} .
\end{aligned}
$$

We can continue these arguments to obtain the following inversion formulas. Given functions $A(j, m)$ and $B(j, m)$ over positive integers $j$ and $m$,

$$
\begin{aligned}
& A(j, m)=\sum_{d \mid m}(1 / d) \sum_{e \mid j ;(e, d)=1} B(j d / e, m / d) \text { if and only if } \\
& B(j, m)=\sum_{d \mid j} \sum_{e \mid m} \mu(d) \mu(e) \sum_{c \mid m / e} c A(j e / d, c) .
\end{aligned}
$$

Given fixed $u$ and $v$ with $(u, v)=1$, if we choose $A(j, m)=\psi_{j}(m u, m v)$ and $B(j, m)=\tau_{j}(m u, m v)$, then (11) produces an exact solution to (3).

\section{REFERENCES}

1. L. Carlitz, The distribution of irreducible polynomials in several indeterminates, Illinois J. Math. 7 (1963), 371-375. MR 27 \#3627.

2. —_ The distribution of irreducible polynomials in several indeterminates. II, Canad. J. Math. 17 (1965), 261-266. MR 30 \#3088.

3. Stephen D. Cohen, The distribution of irreducible polynomials in several indeterminates over a finite field, Proc. Edinburgh Math. Soc. (2) 16 (1968/69), 1-17. MR 38 \#138.

Department of Computer Science, Stanford University, Stanford, California 94305 